\title{
Partitioning-Defective-6-Ephrin-B1 Interaction Is Regulated by Nephrin-Mediated Signal and Is Crucial in Maintaining Slit Diaphragm of Podocyte
}

\author{
Sayuri Takamura, ${ }^{* \dagger}$ Yoshiyasu Fukusumi, ${ }^{*}$ Ying Zhang, ${ }^{*}$ Ichiei Narita, ${ }^{\dagger}$ and Hiroshi Kawachi ${ }^{*}$
}

From the Department of Cell Biology* and the Division of Clinical Nephrology and Rheumatology, ${ }^{\dagger}$ Kidney Research Center, Niigata University Graduate School of Medical and Dental Sciences, Niigata, Japan

\author{
Accepted for publication \\ October 18, 2019. \\ Address correspondence to \\ Hiroshi Kawachi, M.D., Ph.D., \\ Department of Cell Biology, \\ Kidney Research Center, Nii- \\ gata University Graduate \\ School of Medical and Dental \\ Sciences, 1-757 Asahimachi- \\ dori, Chuo-ku, Niigata 951- \\ 8510, Japan. E-mail: \\ kawachi@med.niigata-u.ac.jp.
}

\begin{abstract}
Ephrin-B1 plays a critical role at slit diaphragm. Partitioning-defective (Par)-6 is down-regulated in podocyte of ephrin-B1 knockout mouse, suggesting that Par- 6 is associated with ephrin-B1. Par polarity complex, consisting of Par-6, Par-3, and atypical protein kinase $C$, is essential for tight junction formation. In this study, the expression of Par- 6 was analyzed in the normal and nephrotic syndrome model rats, and the molecular association of Par- 6 , Par-3, ephrin-B1, and nephrin was assessed with the human embryonic kidney 293 cell expression system. Par- 6 was concentrated at slit diaphragm. Par 6 interacted with ephrin-B1 but not with nephrin, and Par-3 interacted with nephrin but not with ephrinB1. The complexes of Par-6-ephrin-B1 and Par-3-nephrin were linked via extracellular sites of ephrinB1 and nephrin. The Par-6-ephrin-B1 complex was delinked from the Par-3-nephrin complex, and Par6 and ephrin-B1 were clearly down-regulated already at early phase of nephrotic model. The alteration of Par-6/ephrin-B1 advanced that of Par-3/nephrin. Stimulation to nephrin phosphorylated not only nephrin but also ephrin-B1, and consequently inhibited the interaction between ephrin-B1 and Par- 6 . Par- 6 appeared at presumptive podocyte of early developmental stage and moved to basal area at capillary loop stage to participate in slit diaphragm formation at the final stage. Par-6-ephrin-B1 interaction is crucial for formation and maintenance of slit diaphragm of podocyte. (Am J Pathol 2020, 190: 333-346; https://doi.org/10.1016/j.ajpath.2019.10.015)
\end{abstract}

A slit diaphragm connecting neighboring foot processes of podocyte is a unique cell-to-cell junction and functions as a final barrier preventing the leak of plasma proteins into primary urine. Proteinuria in several glomerular diseases results from the dysfunction of slit diaphragm, such as minimal change nephrotic syndrome (MCNS), focal segmental glomerulosclerosis (FSGS), and membranous nephropathy. ${ }^{1-}$ Some proteins have been identified as essential molecules of the slit diaphragm, such as nephrin, klin of IRRE-like protein 1 (NEPH1), and podocin. ${ }^{4-6}$ However, the precise molecular composition of the slit diaphragm and the pathogenic mechanism of the slit diaphragm injury are not well understood.

Ephrin and Eph are membrane-bound proteins that function as receptor-ligand pairs. B-type ephrins have a transmembrane domain, followed by a short cytoplasmic region containing four tyrosine residues and a postsynaptic density protein-95/Drosophila discs large/zonula occludens1 (PDZ) domain-binding motif. Ephrin Bs are expressed in several tissues, and ephrin-B plays a critical role in

\footnotetext{
Supported by Ministry of Education, Culture, Sports, Science and Technology of Japan grant-aids for scientific research B: 19H03673 (H.K.) and C: 19K08720 (Y.Z.) and grant-aid for young sciences B: 16K197479 (Y.F.); Astellas Pharma Inc. research aid (H.K.); and Takeda Science Foundation research aid (Y.F.).

Disclosures: None declared.

Portions of this work were presented at the annual meeting of the American Society of Nephrology, on November 2, 2017, New Orleans, LA, and were published in abstract form.
} 
maintaining tissue function in several major organs. ${ }^{7-9}$ It was reported that the overexpression of ephrin-B1 triggered a change in the level of cell-cell adhesion in a study using Madin-Darby canine kidney cells. ${ }^{10}$ Another report showed that the Eph-ephrin-B family affects the cytoarchitecture of tubular epithelial cells and regulates their paracellular permeability. ${ }^{11}$ Ephrin-B1 is restrictedly expressed at the slit diaphragm of podocyte in glomeruli. ${ }^{12}$ Podocyte-specific ephrin-B1 conditional knockout (CKO) mice display the alteration of podocyte morphology, disarrangement of the slit diaphragm molecules, and proteinuria. ${ }^{13}$ The report also showed that ephrin-B1 interacts with nephrin, a key molecule of the slit diaphragm, via their extracellular domains. These findings showed that ephrin-B is essential for maintenance of the slit diaphragm. However, the precise function of ephrin-B1 in podocyte is not fully understood yet.

To better understand the function of ephrin-B 1 at the slit diaphragm, we identified the molecules associated with ephrin-B1 in podocyte. Partitioning-defective (Par)-6 was clearly down-regulated in glomeruli of the podocytespecific ephrin-B1 CKO mice. Cytoskeletal structure was altered in the cultured podocyte in which Par- 6 expression was knocked down. It is reported that ephrin-B regulates tight junctions through the Par complex. ${ }^{14}$ These findings suggested that Par-6 is associated with ephrin-B1 and that Par-6 participates in maintaining podocyte function. Par complex, constituted of Par-6, Par-3, atypical protein kinase $\mathrm{C}(\mathrm{aPKC})$, and cell division cycle 42 (Cdc42), is essential for the establishment of cell polarity and the formation of tight junctions. ${ }^{15-17}$ It is reported that Par-3 is expressed at the slit diaphragm and interacts with the slit diaphragm components. ${ }^{18,19}$ The studies with KO mice showed that aPKC $\lambda$ plays an essential role for maintenance of the slit diaphragm. ${ }^{20-22}$ Par- 6 plays a central role for the formation and the stabilization of the Par complex. ${ }^{15,16}$ However, the expression and function of Par-6 in podocytes are not well elucidated. No studies analyzing the interaction of the Par complex with ephrinB1 in podocytes are reported.

This study showed that Par-6 was concentrated at slit diaphragm. Par- 6 interacted with ephrin-B1 but not with nephrin, and Par-3 interacted with nephrin but not with ephrin-B1. Ephrin-B1 interacts with nephrin via their extracellular site. ${ }^{13}$ Par-6 interacts with Par-3 at cytoplasm. ${ }^{15,16}$ The molecular complex consisting of these four molecules may play a critical role in maintaining slit diaphragm. Herein, we demonstrated that Par-6 and ephrin-B1 were clearly down-regulated already at early phase of nephrotic models. The alteration of Par-6/ephrin-B1 advanced that of Par-3/nephrin. Stimulation to nephrin phosphorylated not only nephrin but also ephrin-B1, and consequently inhibited the interaction of ephrin-B1 with Par-6. Developmental analyses showed that Par-6 moved to basal area of podocyte at the capillary loop stage and participates in slit diaphragm formation at the final stage. It is conceivable that Par-6-ephrin-B1 interaction is crucial for the formation and maintenance of slit diaphragm of podocytes.

\section{Materials and Methods}

\section{Animal Experiments}

All animal experiments conformed to the NIH Guide for the Care and Use of Laboratory Animals. ${ }^{23}$ All procedures in this study were approved by the Animal Committee at Niigata University (Niigata, Japan; permit numbers SA00058 and SA00069). Podocyte-specific conditional knockout mice were prepared, as previously described. ${ }^{13}$ For the experiments with rat, specific pathogen-free, 6-week-old Wister rats were used. For the analyses of developmental expression, kidneys of neonatal Wistar rats were used. Anti-nephrin antibody-induced nephropathy was achieved by a single i.v. injection with $10 \mathrm{mg} / \mathrm{rat}$ of anti-nephrin monoclonal antibody (5-1-6). ${ }^{24-26} \mathrm{~A}$ total of 12 rats were injected with the antibody, and six rats each were killed 1 hour and 5 days after injection. Puromycin aminonucleoside (PAN) nephropathy, a mimic of MCNS, was induced by a single i.v. injection of PAN (100 mg/kg body weight). ${ }^{27}$ Adriamycin (ADR) nephropathy, a mimic of FSGS, was induced by an injection of ADR ( $6 \mathrm{mg} / \mathrm{kg}$ body weight). ${ }^{28}$

\section{Immunohistochemical Analysis}

Immunofluorescence and immunoelectron microscopy were performed basically according to the method previously reported. ${ }^{12,13,26,29,30}$ The primary antibodies used for immunohistochemical analyses were as follows: rabbit anti-Par-6 antibody (Santa Cruz Biotechnology, Dallas, TX), rabbit anti-Par-3 antibody (Merck, Darmstadt, Germany), goat anti-ephrin-B1 antibody (R\&D Systems, Minneapolis, MN), mouse anti-zonula occludens protein 1 antibody (Thermo Fisher Scientific, Waltham, MA), mouse anti-nephrin antibody (monoclonal antibody 5-1-6; prepared in our laboratory), ${ }^{24}$ and rabbit anti-nephrin antibody (prepared in our laboratory). ${ }^{25}$ The immunofluorescence staining in glomeruli was semiquantified basically, according to the method previously reported (score 0 indicates completely absent; 1 , signal covering $0 \%$ to $25 \%$ of the glomerular tuft area; 2 , signal covering $25 \%$ to $50 \%$ of the glomerular tuft area; 3 , signal covering $50 \%$ to $75 \%$ of the glomerular tuft area; and 4, signal covering $75 \%$ to $100 \%$ of the glomerular tuft area). ${ }^{31}$ More than 30 glomeruli of each rat were analyzed.

\section{Immunoprecipitation Assay with Glomerular Lysates}

Immunoprecipitation assay with rat glomerular lysate was performed basically, according to the method reported previously, ${ }^{12}$ with rabbit anti-nephrin antibody, ${ }^{25}$ antiephrin-B1 (R\&D Systems), anti-Par-3 antibody (Merck), or anti-Par-6 antibody (Santa Cruz Biotechnology). Alkaline 

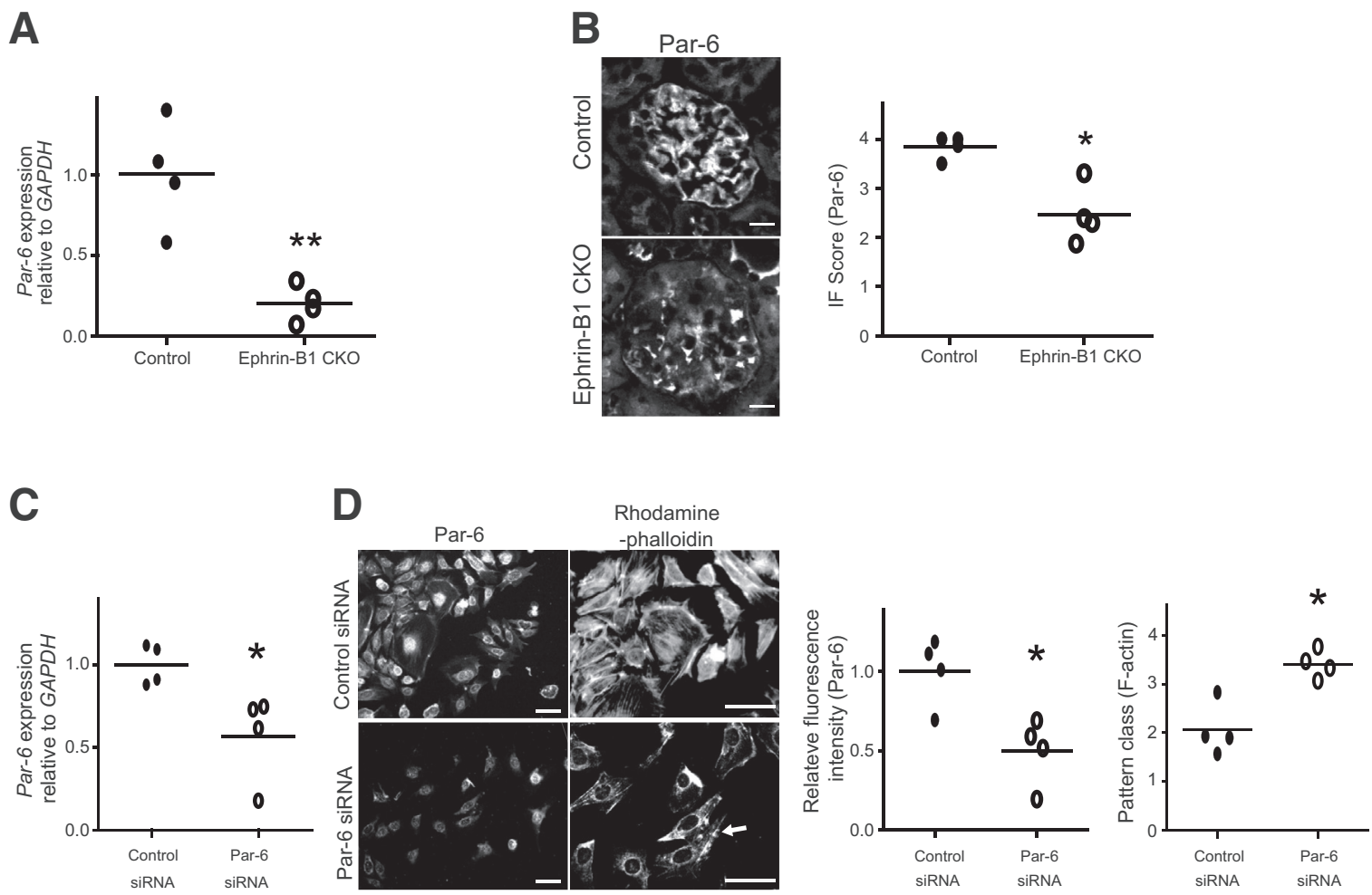

Figure 1 Par-6 expression in the glomeruli of the ephrin-B1 conditional knockout (CK0) mice and finding of Par- 6 knockdown cultured podocyte. A: Realtime PCR analyses: The glomerular mRNA expression of Par- 6 decreases in ephrin-B1 CKO mice $(20 \% \pm 11 \%$; two-tailed $t$-test). B: Immunofluorescence (IF) analyses: Par-6 staining clearly decreases in ephrin-B1 CKO mice (U-test). C: mRNA expression of Par-6 in the cultured podocytes treated with siRNA for 48 hours. The data are shown as the ratio (\%) relative to the control siRNA-treated cells. The expression of Par- 6 decreases to $55.8 \%$ (two-tailed $t$-test). D: F-actin structure, detected with rhodamine-phalloidin, is clearly altered in the cells treated with siRNA for Par- 6 (arrow) $\left(U\right.$-test). ${ }^{*} P<0.05,{ }^{* *} P<0.01$ versus control. Scale bars: $20 \mu \mathrm{m}$ (B); $100 \mu \mathrm{m}$ (D).

phosphatase-conjugated anti-rabbit IgG (Thermo Fisher Scientific) and alkaline phosphatase-conjugated anti-goat IgG (Santa Cruz) were used as secondary antibodies. The reaction was developed with an alkaline phosphatase chromogen kit (Kirkegaard \& Perry Laboratories, Inc., Gaithersburg, MD).

\section{Assays with HEK293 Cell Transfection}

Assays with human embryonic kidney (HEK) 293 cell transfection were performed, as previously described. ${ }^{13}$ HEK293 cells were transfected with pk-myc-tagged Par-6 (Addgene, Cambridge, MA; plasmid number 15473), pk-myc-tagged Par-3 (Addgene; plasmid number 19388), HA-tagged ephrin-B1, or FLAG-tagged nephrin by calcium phosphate method. pk-myc-Par-6 and pk-myc-Par-3 were gifts from Dr. Ian Macara (University of Virginia Health Sciences Center, Charlottesville, VA). ${ }^{15}$ HA-ephrin-B1 and FLAG-nephrin were prepared, as previously described. ${ }^{13}$ After the transfection, cells were lysed with $1 \%$ Triton in Tris- $\mathrm{HCl}$ buffer with proteinase inhibitors and $1 \mathrm{mmol} / \mathrm{L}$ sodium vanadate. The lysates were incubated with rabbit anti-FLAG antibody (Sigma-Aldrich, St. Louis, MO), mouse anti-FLAG antibody (Sigma-Aldrich), rabbit anti-HA antibody (Bethyl Laboratories, Montgomery, TX), mouse
anti-HA antibody (Santa Cruz Biotechnology), rabbit antimyc antibody (MBL, Woburn, MA), mouse anti-myc antibody (Santa Cruz Biotechnology), or anti-Par3 antibody (Merck) at $4{ }^{\circ} \mathrm{C}$ overnight and precipitated with Dynabeads Protein G (Invitrogen, Carlsbad, CA).

For in vitro phosphorylation assay, the transfected HEK293 cells were treated with $50 \mu \mathrm{mol} / \mathrm{L}$ pervanadate (protein tyrosine phosphatase inhibitors) for 10 minutes. After treatments, cells were stimulated with $50 \mu \mathrm{g} / \mathrm{mL}$ of mouse anti-nephrin antibody ${ }^{24}$ or $2 \mu \mathrm{g} / \mathrm{mL}$ of EphB2-Fc (R\&D Systems) for 10 minutes. After the stimulation, cells were lysed with lysing buffer with $1 \mathrm{mmol} / \mathrm{L}$ sodium vanadate.

\section{Real-Time RT-PCR}

Real-time RT-PCR analyses were performed according to the method described previously. ${ }^{13,30,32}$ The glomeruli of ephrinB1 CKO mice were isolated, according to the method previously reported. ${ }^{33}$ The glomeruli were homogenized, and then total RNA was extracted. PCR was performed with the following mouse Par-6 primers: sense, 5'-TGGGCAC-

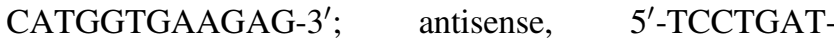
CAGGGTGTCCGTGC- $3^{\prime}$. The data are shown as ratios relative to control findings and expressed as means \pm SD of four independent experiments. 
A

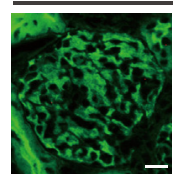

B

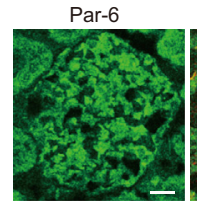

C

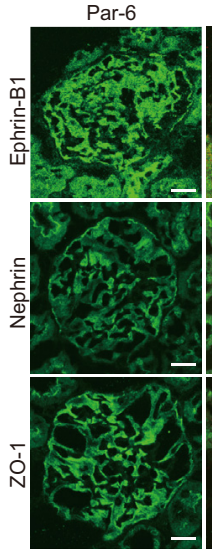

Par-3
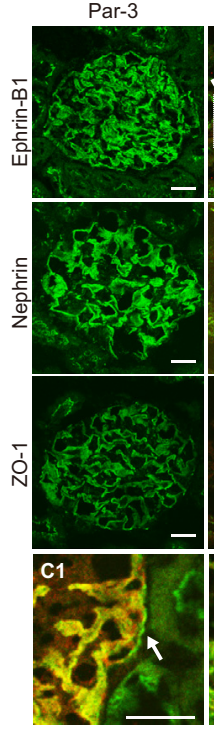

NRS
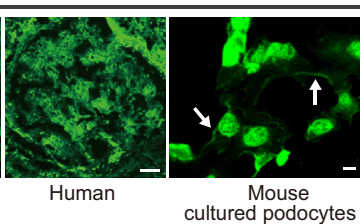

Merge

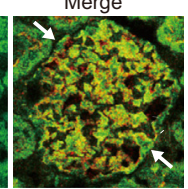

Par-3

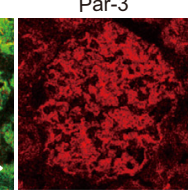

Merge

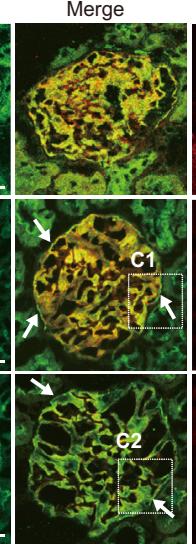

Marker

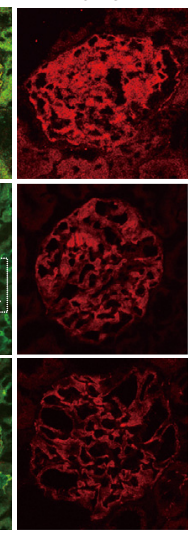

Merge

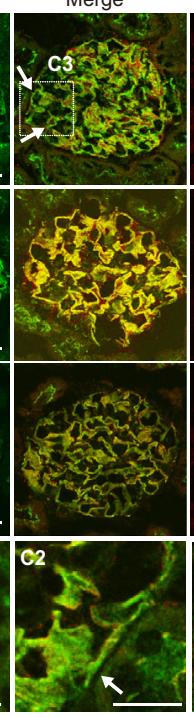

Marker

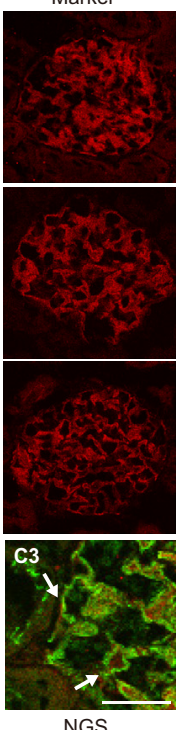

NGS
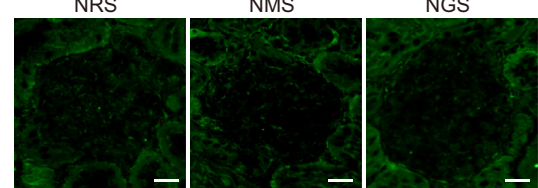

D

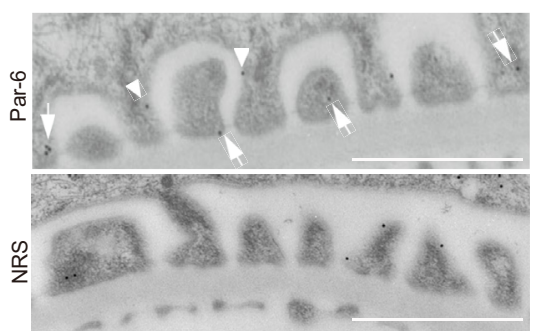

\section{RNA Silencing Analysis}

A conditionally immortalized mouse podocyte cell line was kindly donated by Dr. Peter Mundel (Albert Einstein College of Medicine, Bronx, NY). Cells were cultivated, as previously described. ${ }^{32,34}$ The differentiated cells showing the prominent processes and mRNA expression of the differentiated molecules nephrin and podocin were used for the experiments. The siRNA targeting Par-6 and negative control siRNA were purchased from Qiagen Inc. (Valencia, CA). Cells were harvested for 48 hours after siRNA treatment for real-time RT-PCR analyses and immunofluorescence. Fluorescence intensity of Par- 6 was evaluated with ImageJ software version 1.47 (NIH, Bethesda, MD; https:// imagej.nih.gov/ij). The area of individual cells was outlined manually. Fluorescence intensity in the outlined area was recorded. Cellular immunofluorescence intensities were normalized for cell area. The immunofluorescence intensity was assigned to each cell, and 30 cells of each image were analyzed. Actin staining was evaluated, as previously described. ${ }^{35}$ To detect actin fiber, rhodaminephalloidin (Cytoskeleton Inc., Denver, CO) was used.

\section{Statistical Analysis}

Statistical significance was evaluated using the unpaired $t$-test or the $U$-test. Data were analyzed using GraphPad Prism 5.0 software version 5.04 (GraphPad Software, San Diego, CA).

\section{Results}

Par-6 Is Down-Regulated in Glomeruli of the PodocyteSpecific Ephrin-B1 CKO Mice, and F-Actin Structure Is Altered in Par-6 Knockdown Podocytes

Because RNA-sequencing analysis with glomerular sample showed that mRNA expression of Par-6 of the podocytespecific ephrin-B1 CKO mice was down-regulated to almost $50 \%$ comparing with wild-type control mice, the expression

Figure 2 Localization of Par-6 and Par-3 in glomeruli. A: Par- 6 staining is detected along the capillary loop in rat and human glomeruli. Par-6 staining is detected at periphery of the mouse cultured podocytes (arrows). B: Dual-labeling findings of Par-6 and Par3. Par-6 staining is detected more broadly than Par-3 (arrows). C: Dual-labeling findings of Par-6 and Par-3 with ephrin-B1, nephrin, and zonula occludens protein 1 (Z0-1). Par-6 colocalizes with ephrin-B1 almost completely. Some portions of the Par- 6 staining are apart from nephrin and Z0-1 (arrows in Z0-1). Par3 colocalizes with nephrin, and some portions of the Par-3 staining are apart from ephrin-B1 (arrows in ephrin-B1). Par-3 colocalizes with Z0-1. Negative findings with normal rabbit serum (NRS), normal mouse serum (NMS), and normal goat serum (NGS) are shown at the bottom. Boxed areas are shown at higher magnification in the corresponding panel below (C1-C3). D: Immunoelectron microscopic finding of Par-6. Gold particles for Par- 6 are mainly detected at the slit diaphragm (arrows), and some particles are detected just above the slit diaphragm (arrow heads). Almost negative finding is detected with NRS. Scale bars: $20 \mu \mathrm{m}(\mathbf{A}-\mathbf{C}) ; 1 \mu \mathrm{m}$ (D). 
of Par-6 in the CKO mice was analyzed. Real-time PCR analysis showed that the mRNA expression of Par-6 decreased to $20.0 \% \pm 11.0 \%$ for control mice $(P<0.01$, two-tailed $t$-test) (Figure 1A). The immunohistochemical analysis also showed that the expression of Par-6 was clearly decreased in the CKO mice (staining score for CKO mice versus control mice, $2.47 \pm 0.60$ versus $3.84 \pm 0.24$; $P<0.05 ; U$-test) (Figure 1B). These findings suggested that Par-6 was associated with ephrin-B1 in podocytes. To presume the role of Par- 6 in podocytes, the phenotype of the cultured podocyte in which the expression of Par-6 was
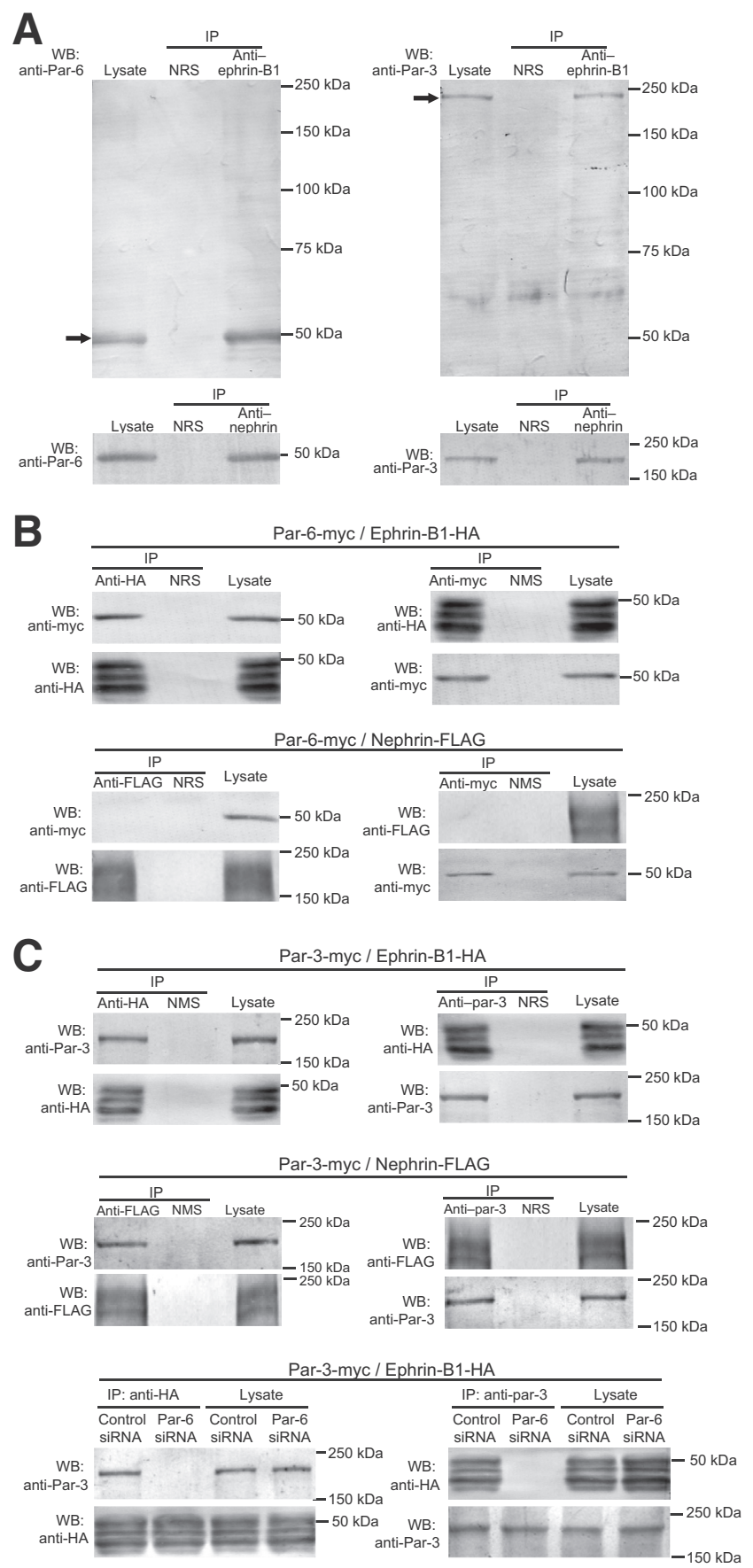

knocked down by siRNA treatment was analyzed. The efficiency of siRNA treatment is confirmed by real-time PCR analysis (average value of decrease, 55.8\%; $P<0.05$ ) (Figure 1C). Par-6 staining in cultured podocytes was evaluated with ImageJ (versus control siRNA: $0.50 \pm 0.21$ versus $1.00 \pm 0.22$ ) (Figure 1D). F-actin structure, detected with rhodamine-phalloidin, was clearly altered in the cells treated with siRNA for Par-6 (category of F-actin distribution for Par-6 siRNA versus control siRNA, $3.41 \pm 0.29$ versus $2.06 \pm 0.54 ; P<0.05$ ) (Figure $1 \mathrm{D}$ ). The result suggested that Par- 6 participates in the maintenance of the specialized podocyte morphology.

\section{Par-6 Colocalizes with Ephrin-B1 at Slit Diaphragm, and Par-3 Colocalizes with Nephrin}

Next, the localization of Par- 6 in glomeruli was analyzed. Par-6 was detected along glomerular capillary wall in normal rat and human kidney sections (Figure 2A). In normal rat kidney section, Par-6 was detected more broadly than Par-3 (Figure 2B). Par-6 was almost completely costained with ephrin-B1. On the other hand, Par-3 was almost completely costained with nephrin. The localizations of Par6 and Par-3 were compared with that of zonula occludens protein 1 . Par-6 located slightly aside from zonula occludens protein 1, whereas the staining of Par-3 almost completely coincided with zonula occludens protein 1 (Figure 2C). Immunoelectron microscopic analysis showed Par-6 was mainly expressed at slit diaphragm and was also detected at cell surface just above slit diaphragm (Figure 2D).

Figure 3 Par-6 interacts with ephrin-B1 but not with nephrin, and Par-3 interacts with nephrin but not with ephrin-B1. A: Interaction assays with rat glomerular lysates. A specific Par- 6 band of approximately $50 \mathrm{kDa}$ is detected in the precipitates with anti-ephrin-B1 antibody (arrow, left panel), and a specific Par-3 band of approximate $180 \mathrm{kDa}$ is detected in the precipitates with anti-ephrin-B1 antibody (arrow, right panel). B: Interaction assays of Par-6 with ephrin-B1 and nephrin with the lysates of human embryonic kidney (HEK) 293 cells cotransfected with Par-6-myc and ephrin-B1-HA (top panel) and with Par-6-myc and nephrin-FLAG (bottom panel). The Par-6 band is detected in the precipitates with the anti-HA antibody, and the ephrinB1 band is detected in precipitates with anti-myc antibody. The Par- 6 band is not detected in the precipitates with anti-FLAG antibody, and the nephrin band is not detected in the precipitates with anti-myc antibody. C: Top panel: Interaction assays of Par-3 with ephrin-B1 and nephrin. The assay with the lysates of HEK293 cells cotransfected with Par-3-myc and ephrin-B1-HA. The Par-3 band is detected in the precipitate with anti-HA. The ephrin-B1 band is detected in the precipitate with anti-Par-3. Middle panel: The assay with the lysates of HEK293 cells cotransfected with Par-3-myc and nephrin-FLAG. The Par-3 band is detected in the precipitate with anti-FLAG. The nephrin band is detected in the precipitates with anti-Par-3 antibody. Bottom panel: Interaction assays of Par-3 with ephrin-B1 with HEK293 cells treated with siRNA for Par-6. In the lysates of the HEK293 cells treated with siRNA for Par-6, the Par- 3 band was not detected in the precipitates with anti-HA antibody and the ephrin-B1 band was not detected in the precipitates with anti-Par-3 antibody. IP, immunoprecipitation; NMS, normal mouse serum; NRS, normal rabbit serum; WB, Western blot. 
Par-6 Interacts with Ephrin-B1 but Not with Nephrin, and Par-3 Interacts with Nephrin but Not with Ephrin-B1

In the immunoprecipitation assay with normal rat glomerular lysate, both Par- 6 and Par-3 bands were detected in the precipitate with anti-ephrin-B1 antibody. Both Par-6 and Par-3 bands were detected also in the precipitate with anti-nephrin antibody (Figure 3A). The assay with the HEK cell expression system showed that Par-6 interacted with ephrin-B1, but not with nephrin (Figure 3B). The assay also showed that Par-3 interacted with both ephrin-B1 and nephrin (Figure 3C). However, because HEK293 cells highly expressed Par-6, it was verified whether the binding of Par-3 with ephrin-B1 was mediated with endogenous Par-6 of HEK293 cells. The interaction of Par-3 with ephrin-B1 was analyzed in the HEK293 cells in which endogenous Par-6 was knocked down with siRNA. The interaction of Par-3 and ephrin-B1 was not detected in the Par-6 knockdown HEK293 cells (Figure 3C). These results showed that Par-6 interacts with ephrin-B1 and that Par-3 interacts with nephrin. Ephrin-B1 was also shown to interact with nephrin. It is conceivable that the Par-6-ephrinB1 complex linked to the Par-3-nephrin complex via ephrinB1-nephrin interaction, and that the complex consisting of these four molecules is a critical structure of slit diaphragm.
Par-6 and Ephrin-B1 Are Clearly Down-Regulated at the Early Phase of the Anti-Nephrin Antibody-Induced Nephropathy, and Par-3 and Nephrin Are Down-Regulated at the Proteinuric Phase

The expression of Par- 6 and Par-3 was next examined in the slit diaphragm dysfunction in the rat nephrotic model caused by the anti-nephrin antibody injection. ${ }^{24-26} \mathrm{~A}$ single i.v. injection of the anti-nephrin antibody caused transient but massive proteinuria in rats. Proteinuria is induced without participation of any inflammatory factors ${ }^{36}$ and is caused by disarrangement of the slit diaphragm components..$^{25,37,38}$ The nephropathy was not accompanied with any morphologic alterations. In this nephropathy, proteinuria occurs within 24 hours after the antibody injection and becomes the peak at the 5th to 8th day, and then decreases to normal level by the 18th day. The expression of Par- 6 was clearly decreased at 1 hour after the anti-nephrin antibody injection (staining score versus normal rat, $1.65 \pm 0.57$ versus $3.76 \pm 0.21 ; P<0.01 ; U$-test). The expression of Par- 6 recovered to almost normal level on day 5 when proteinuria was still evident $(138.9 \pm 51.0 \mathrm{mg} /$ day). On the other hand, the decrease in the expression of Par3 was not remarkable at the early phase, but the expression of Par-3 was clearly decreased on day 5 (staining score,
A
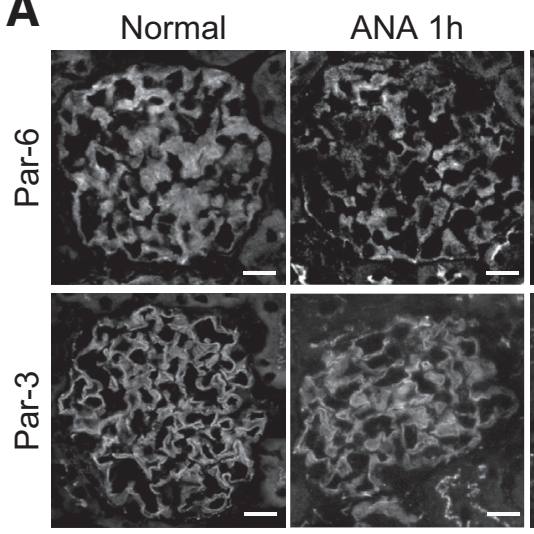

B
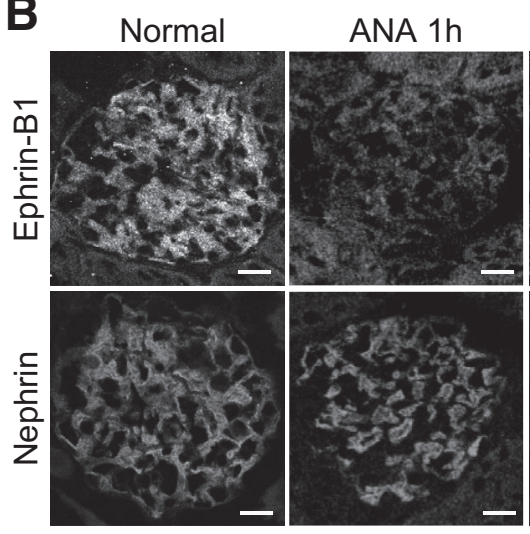

ANA Day 5
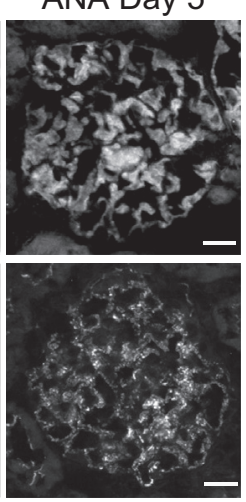

ANA Day 5

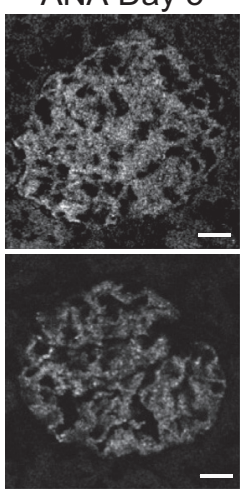

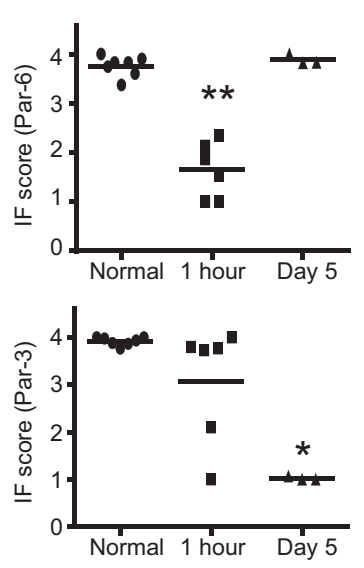

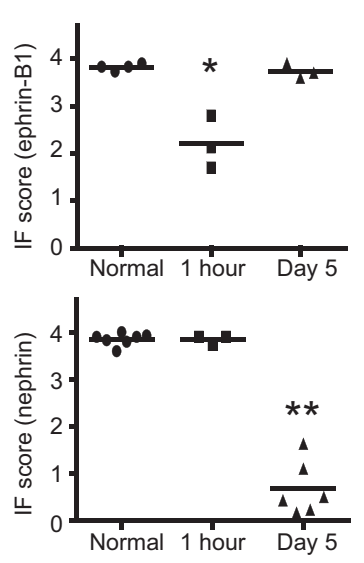

Figure 4 Expression of Par-6 and Par-3 in the anti-nephrin antibody-induced nephropathy (ANA). A: Par-6 expression is clearly decreased at 1 hour. The expression of Par- 6 recovers on day 5 when proteinuria is still detected. The expression of Par-3 is not evidently altered at 1 hour, and Par-3 expression clearly decreases on day 5. B: Ephrin-B1 staining clearly decreases at 1 hour and recovers on day 5. Nephrin staining is not evidently altered at 1 hour. The intensity of nephrin staining clearly decreases, and the staining pattern shifts to discontinuous granular pattern on day $5 .{ }^{*} P<0.05,{ }^{* *} P<0.01$ versus normal ( $U$-test). Scale bars $=20 \mu \mathrm{m}(\mathbf{A}$ and $\mathbf{B})$. IF, immunofluorescence. 

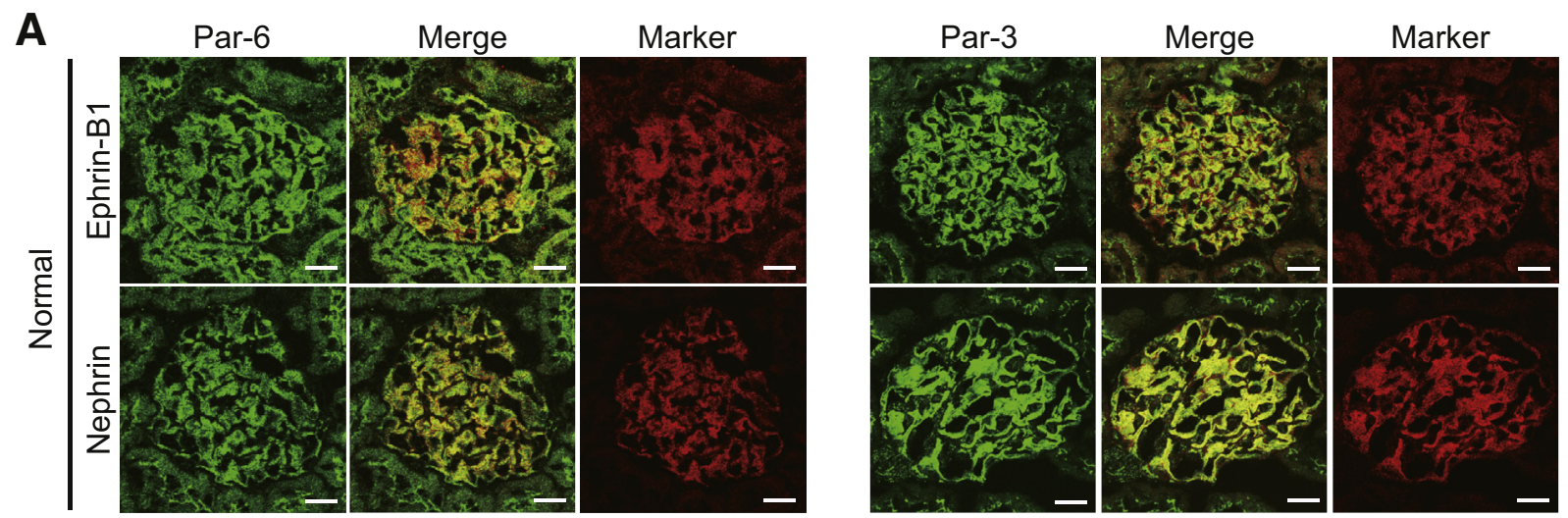

B
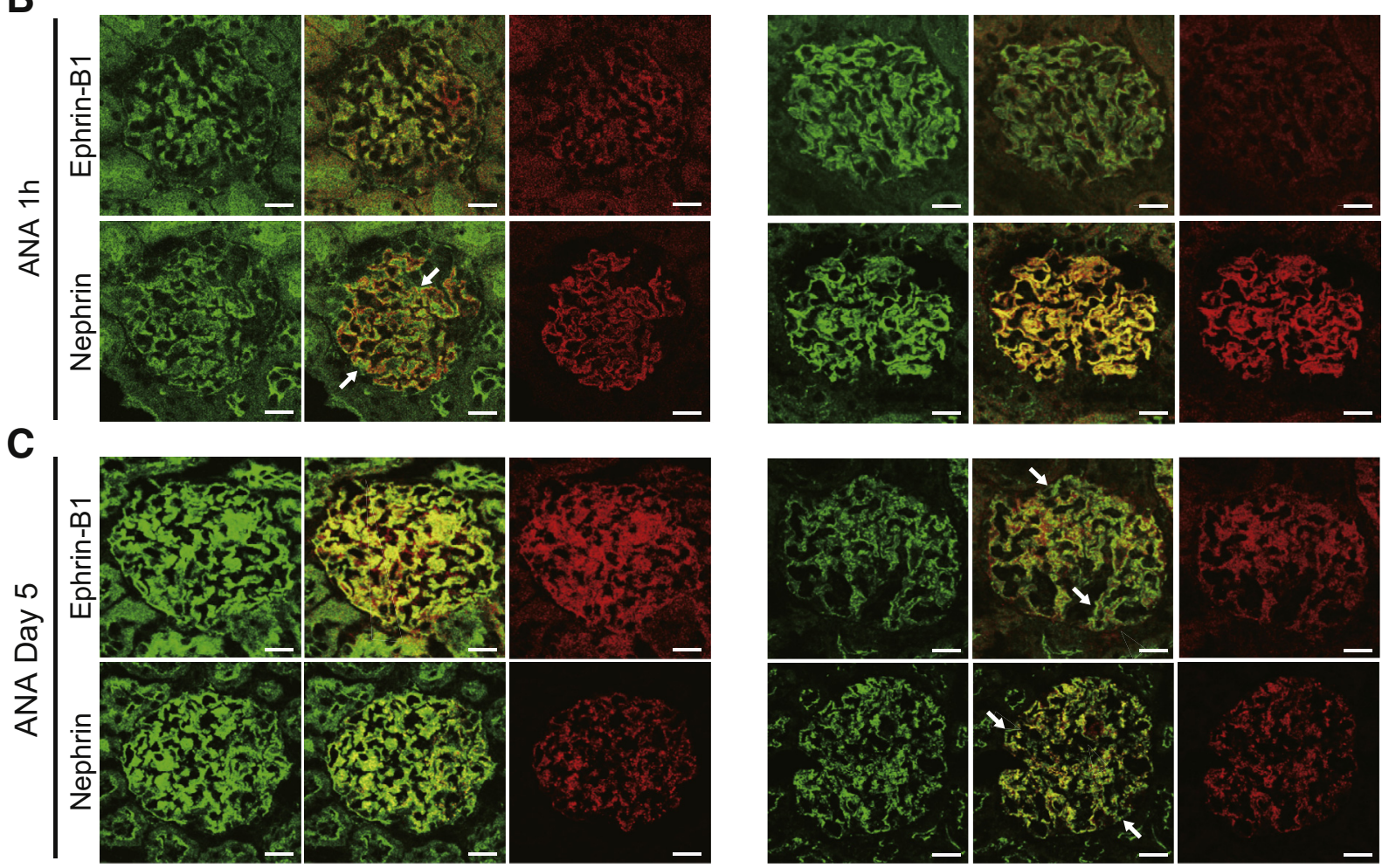

D

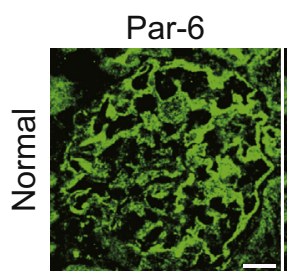

Merge

Par-3
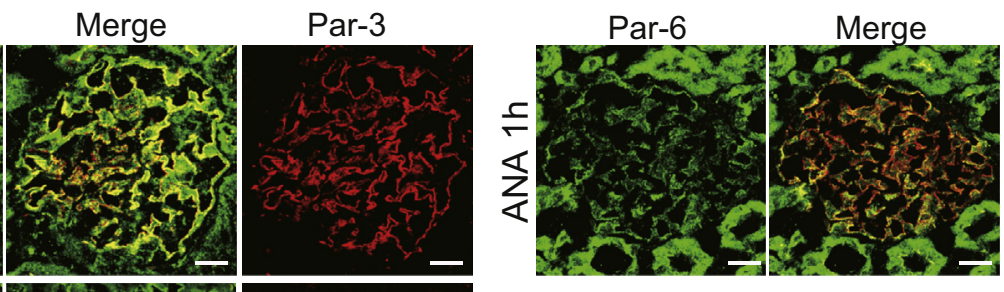

Par-3

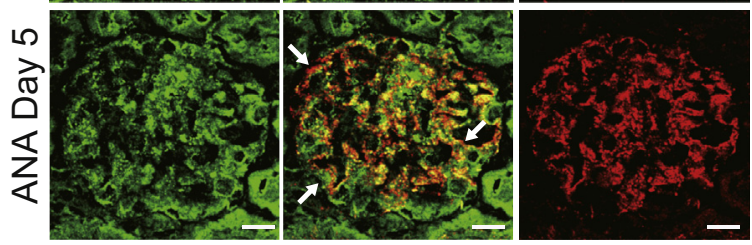

Figure 5 Dual-labeling immunofluorescence findings of Par-6 and Par-3 with ephrin-B1 and nephrin in the anti-nephrin antibody-induced nephropathy (ANA). A: Par-6 almost completely colocalizes with ephrin-B1, and Par-3 colocalizes with nephrin in normal rat glomeruli. B: At 1 hour in ANA, Par-6 and ephrin-B1 staining is clearly lowered. The remaining Par-6 staining is clearly apart from the nephrin staining (arrows). The staining of Par-3 and nephrin is not evidently altered, and they colocalize. C: 0 n day 5, Par- 6 and ephrin-B1 staining recovers, and Par- 6 colocalizes with ephrin-B1. Par-3 and nephrin staining are clearly lowered, and the remaining Par-3 is apart from ephrin-B1 (arrows) and nephrin (arrows). D: 0n day 5, Par-6 staining is apart from the remaining Par-3 (arrows). Scale bar $=20 \mu \mathrm{m}(\mathbf{A}-\mathbf{D})$. 
$1.02 \pm 0.04$ versus $3.92 \pm 0.08 ; P<0.05$ ) (Figure $4 \mathrm{~A}$ ). The ephrin-B1 staining clearly decreased at 1 hour $(2.21 \pm 0.55$ versus $3.82 \pm 0.07 ; P<0.05$ ) (Figure $4 \mathrm{~A}$ ), and it recovered on day 5. By contrast, the alterations in nephrin staining are not clear at 1 hour, and the staining intensity of nephrin clearly decreased on day $5(0.68 \pm 0.57$ versus $3.8 \pm 0.13 ; P<0.01)$ (Figure 4B). The findings indicated that the alteration of Par-6 was parallel to that of ephrin-B1, the alteration of Par-3 was parallel to that of nephrin, and the alteration of Par-6/ephrinB1 advanced that of Par-3/nephrin.

\section{Complex Consisting of Par-6, Par-3, Ephrin-B1, and} Nephrin Breaks in Anti-Nephrin Antibody-Induced Nephropathy

In normal rat kidney section, Par-6 was almost completely colocalized with ephrin-B1, and Par-3 was colocalized with nephrin (Figure 5A). Par-6 and ephrin-B1 staining was
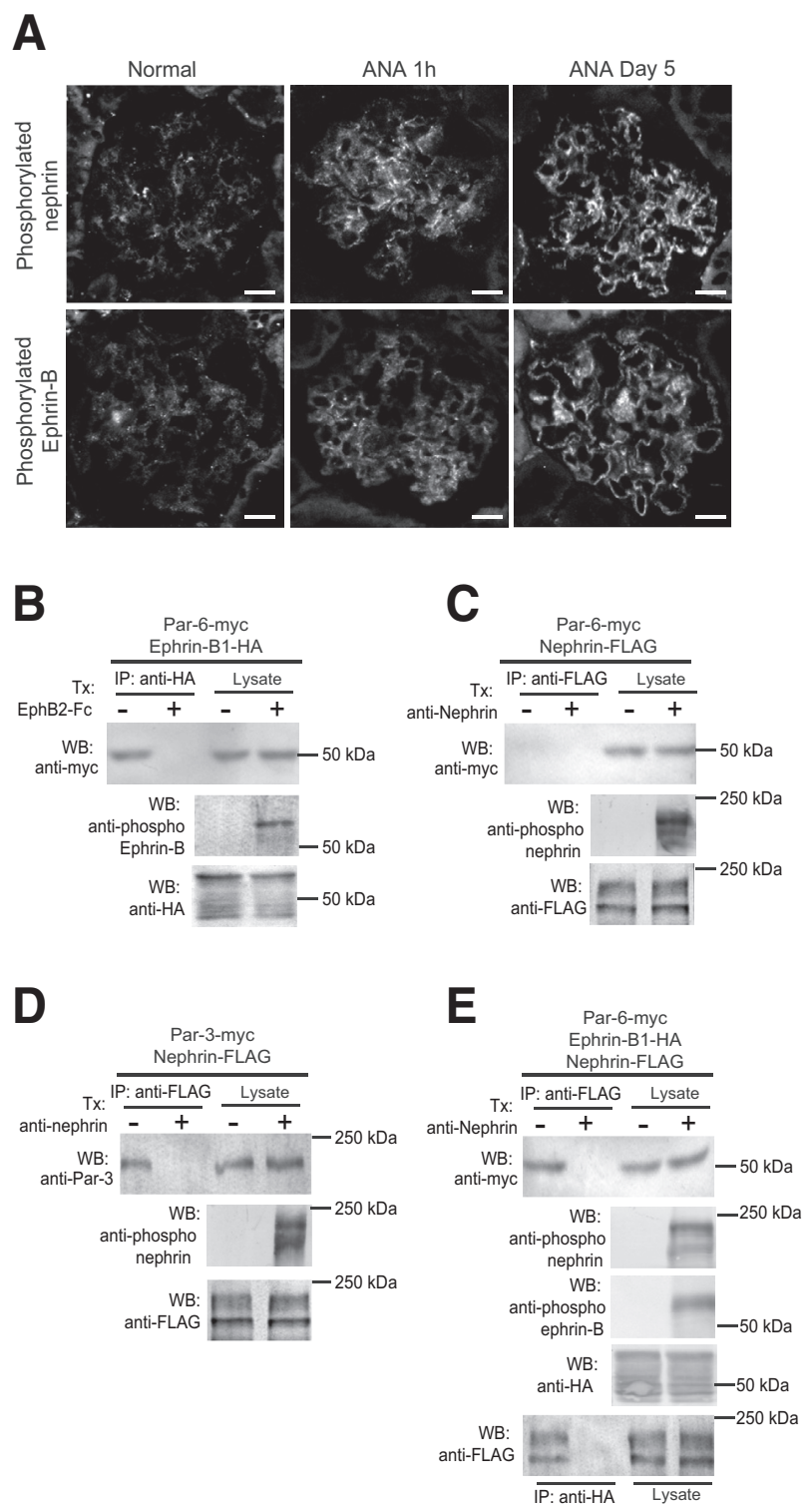

clearly lowered at 1 hour in the anti-nephrin antibody-induced nephropathy. Dual-labeling immunofluorescence analysis showed the remaining Par- 6 was clearly apart from nephrin, although the Par-6 was observed to be colocalized with ephrin-B1 (Figure 5B). On day 5, the Par-6 and ephrin-B1 staining recovered. Although the expression of Par-3 and nephrin was not evidently altered at 1 hour, the expression was clearly lowered on day 5 . The remaining Par-3 was apart from the remaining nephrin (Figure 5C). On day 5, major parts of Par-6 staining were apart from Par-3 staining (Figure 5D). The results imply that the Par-6/ ephrin-B1 complex and the Par-3/nephrin complex were delinked already at the initiation phase of the nephrotic model and that the molecular complex consisting of four molecules breaks at the nephrotic phase.

\section{Par-6 Does Not Interact with the Phosphorylated Ephrin-B1, and Par-3 Does Not Interact with the Phosphorylated Nephrin}

Nephrin and ephrin-B1 in glomeruli were phosphorylated in the anti-nephrin antibody-induced nephropathy (Figure 6A). Next, it was analyzed whether the phosphorylation of ephrin-B1 and/or nephrin affects their interactions with Par-6 and Par-3. The analyses with the HEK cells dual transfected with Par-6 and ephrin-B1 showed that Par-6 did not interact with the ephrin-B1 phosphorylated by the

Figure 6 Par- 6 does not interact with phosphorylated ephrin-B1, and Par-3 does not interact with phosphorylated nephrin. A: Immunofluorescence findings of the phosphorylated nephrin and the phosphorylated ephrin-B1 in the rat kidney sections of the anti-nephrin antibody-induced nephropathy (ANA). The phosphorylation of nephrin and ephrin-B1 is already detected at 1 hour, and the staining with phosphorylated nephrin and phosphorylated ephrin-B antibodies is still positive on day 5. B: Human embryonic kidney (HEK) 293 cells cotransfected with Par-6-myc and ephrinB1-HA were treated with EphB2-Fc. Ephrin-B1 was phosphorylated by the EphB2-Fc treatment (Tx). Par-6 band is detected in the precipitate with anti-HA antibody from the lysates of the HEK293 cell without treatment of EphB2-Fc, but Par- 6 band is not detected from the lysates of the HEK cell treated with EphB2-Fc. C: HEK293 cells cotransfected with Par-6-myc and nephrin-FLAG were treated with the anti-nephrin antibody. Nephrin was phosphorylated by the treatment with the anti-nephrin antibody. Par- 6 band is not detected in the precipitates with anti-FLAG antibody of the HEK293 cell lysates without treatment of anti-nephrin antibody. The Par- 6 band is not detected in HEK293 cell lysates treated with anti-nephrin antibody either. D: HEK293 cells cotransfected with Par-3-myc and nephrin-FLAG were treated with the anti-nephrin antibody. Par-3 band is detected in the precipitate with anti-FLAG antibody of the lysates of the HEK cell without treatment of the anti-nephrin antibody. The Par-3 band is not detected in the precipitate of the lysates of the HEK cell treated with the anti-nephrin antibody. E: HEK293 cells cotransfected with Par-6-myc, ephrin-B1-HA, and nephrin-FLAG were treated with anti-nephrin antibody. Both nephrin and ephrin-B1 are phosphorylated by the anti-nephrin antibody treatment. The Par- 6 band and the nephrin band are detected in the precipitate with the anti-HA antibody of the lysates of the HEK cell without treatment of anti-nephrin antibody. Neither Par- 6 nor nephrin bands are detected in the precipitate with the anti-HA antibody of the lysates of the HEK cell treated with the anti-nephrin antibody. Scale bars $=20 \mu \mathrm{m}(\mathbf{A})$. IP, immunoprecipitation; phospho, phosphorylated; WB, Western blot. 
stimulation with EphB2 (Figure 6B). Par-6 did not interact with nephrin, even if nephrin was phosphorylated (Figure 6C). Par-3 interacted with nephrin, which was not stimulated with anti-nephrin antibody. But if nephrin was

A
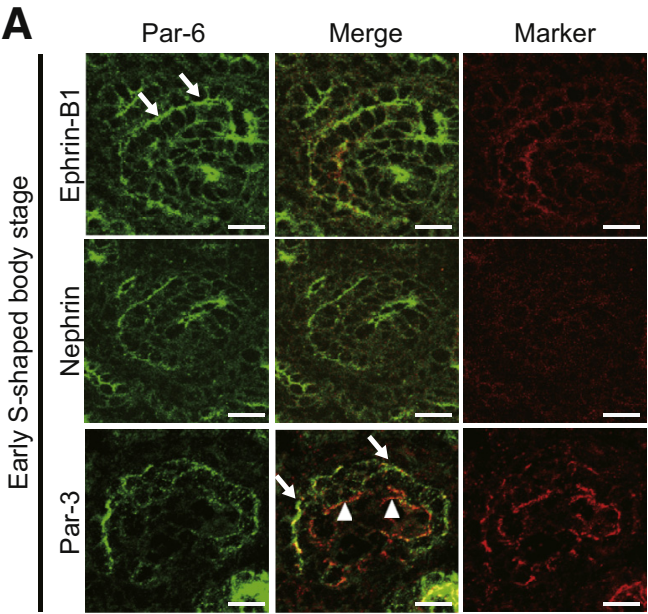

B
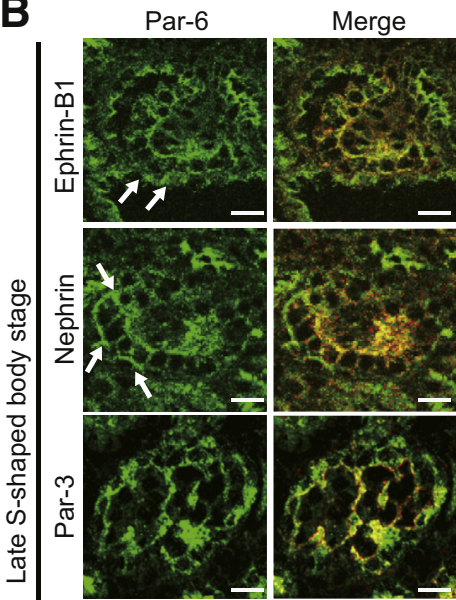

Marker

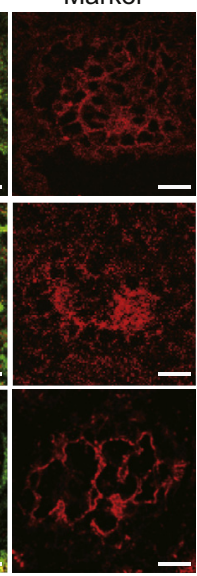

Par-3

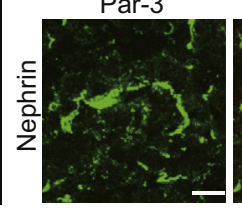

Merge

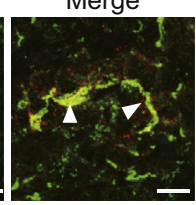

Marker

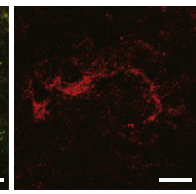

\section{c}

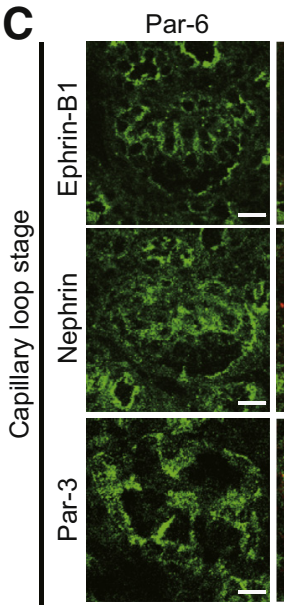

Merge

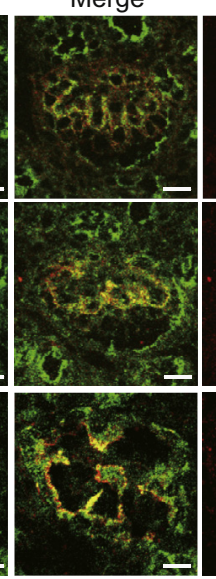

Marker

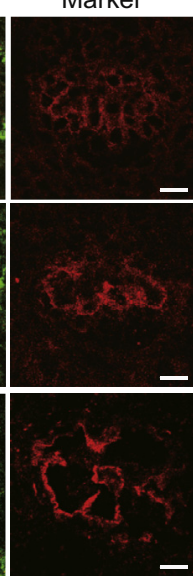

phosphorylated by the stimulation with anti-nephrin antibody, Par-3 did not interact with nephrin (Figure 6D). The analyses with the HEK cells triple transfected with Par-6, ephrin-B1, and nephrin showed that the anti-nephrin antibody binding phosphorylated not only nephrin but also ephrin-B1. The phosphorylated ephrin-B1 did not interact with Par-6 or nephrin (Figure 6E). These findings implied that stimulation to nephrin phosphorylated not only nephrin but also ephrin-B1, and consequently inhibited the interaction of ephrin-B1 with Par-6 and nephrin.

Par-6 Appears in Presumptive Podocyte of Early SShaped Body Stage before the Appearance of Nephrin, and Par-6 Is Restricted to Basolateral Area at the Final Stage of the Podocyte Maturation

The developmental expression of Par-6 in glomeruli was analyzed with neonatal rat kidney sections. Par-6 was expressed in presumptive podocytes of early S-shaped body stage, when nephrin did not appear yet and ephrinB1 staining was faint. At this stage, Par-6 was mainly detected at apical area. Par-3 also appeared in podocyte of early S-shaped body stage. At this stage, Par-3 was mainly detected at basal area of presumptive podocytes and was not costained with Par-6 (Figure 7A). At late S-shaped body stage, ephrin-B1 and nephrin were detected at basolateral surface of podocytes, and Par- 6 was broadly detected along cell surface. At this stage, Par-3 was costained with nephrin (Figure 7B). At capillary loop stage, Par-6 was restrictedly detected at basal area, and major parts of Par-6 were costained with ephrin-B1, nephrin, and Par-3 (Figure 7C). These findings implied that Par-6 moved to basal area and met ephrin-B1, nephrin, and Par-3 at capillary loop stage.

\section{Down-Regulation of Par-6 Is Prolonged in the Nephrotic Models, Mimics of MCNS and FSGS}

Proteinuria in PAN nephropathy caused by a single injection with PAN occurs on day 3 to 5, peaks on around day 10 (day 10, $313 \pm 105 \mathrm{mg} /$ day), and then decreases to normal level within 1 month. ${ }^{28}$ PAN nephropathy is accepted to be an experimental model of human MCNS because the

Figure 7 Developmental expression of Par-6 in glomeruli. A: Early Sshaped body stage: Nephrin staining is not detected, and ephrin-B1 staining is faint. Par-6 is mainly expressed at apical surface (arrows). Par-3 is detected at the basal area of the presumptive podocytes (arrowheads). Par- 6 does not colocalize with Par-3. B: Late S-shaped body stage: Ephrin-B1 and nephrin are detected at basolateral surface. Par- 6 is detected broadly along almost whole cell surface. Although Par- 6 at basolateral area colocalizes with nephrin, ephrin-B1, and Par-3, Par-6 is detected also at apical area (arrows). At this stage, Par-3 almost completely costains with nephrin (arrowheads). C: Capillary loop stage: Par 6 is restrictedly detected at basal area along capillary loop, and major parts of Par- 6 are costained with ephrin-B1, nephrin, and Par-3. Scale bars $=20$ $\mu \mathrm{m}(\mathbf{A}-\mathbf{C})$. 


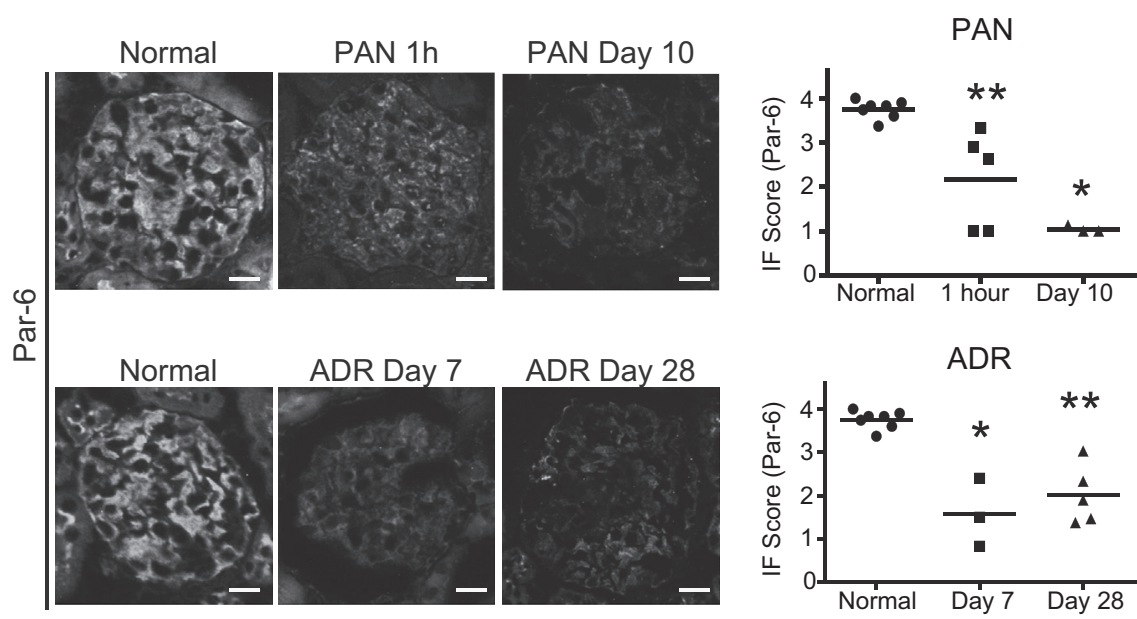

Figure 8 Expression of Par-6 in puromycin aminonucleoside (PAN) and adriamycin (ADR) nephropathy. Representative immunofluorescence (IF) findings and the data from semiquantitative analysis of the staining. Par- 6 staining is clearly decreased at the early phase of PAN (1 hour) and ADR nephropathy (day 7). Par-6 staining does not recover at the proteinuric phase in PAN and ADR nephropathy. ${ }^{*} P<0.05,{ }^{*} P<0.01$ versus normal $(U$-test). Scale bars $=20 \mu \mathrm{m}$. nephropathy was accompanied with severe foot process effacement. ${ }^{39}$ Proteinuria in ADR nephropathy is first detected around day 10 and increases with time (on day 28, $146 \pm 37.5 \mathrm{mg} /$ day). ${ }^{28,40}$ Several morphologic alterations, such as mesangial matrix expansion, foot process effacement, and thickening of glomerular basement membrane, were detected on day 28 in ADR nephropathy. ${ }^{28,40}$ ADR nephropathy is accepted to be an experimental model of FSGS. Decrease in the immunostaining of Par-6 at the initiation phase was also detected in PAN nephropathy (staining score versus control, $2.17 \pm 1.1$. versus $3.75 \pm 0.21 ; P<0.01 ; U$-test $)$ and in ADR nephropathy $(1.58 \pm 0.79 ; P<0.05)$ (Figure 8$)$. The findings implied that the decrease in the expression of Par- 6 could be an early sensitive marker to detect slit diaphragm injury. The decrease of Par-6 staining was still evident on day 10 of PAN nephropathy (staining score, $1.04 \pm 0.77 ; P<0.05$ ) and on day 28 of ADR nephropathy (2.02 \pm 0.68 ; $P<0.01$ ), although the Par-6 staining was recovered on day 5 in the anti-nephrin antibody-induced nephropathy (Figure 4), The observation suggested that monitoring of Par-6 expression may be a useful tool to understand the type and degree of podocyte injury.

\section{Discussion}

In this study, it was first demonstrated that Par-6 is mainly expressed at slit diaphragm and is also expressed at cell surface just above the slit diaphragm (Figure 2). Par-6 almost completely colocalized with ephrin-B1, and was slightly apart from nephrin. On the other hand, Par-3 was almost completely colocalized with nephrin and some portions of Par-3 were apart from ephrin-B1. The interactions of Par-6 and Par-3 with ephrin-B1 and nephrin were then analyzed. In the assay with glomerular lysate, both Par-6 and Par-3 bands were detected in the precipitate with antiephrin-B1 antibody, and both Par-6 and Par-3 bands were detected also in the precipitate with anti-nephrin antibody (Figure 3). The results clearly showed that Par-6 as well as Par-3 is a member of the slit diaphragm complex. The analyses with the HEK cell expression system revealed that Par-6 interacts with ephrin-B1 but not with nephrin, and that Par-3 interacts with nephrin but not with ephrin-B1. EphrinB1 interacts with nephrin via their extracellular portions. ${ }^{13}$ Par-6 interacts with Par-3 via their PDZ domains. ${ }^{15}$ Taken together, it can be concluded that the Par-6-ephrin-B1 complex links to the Par-3-nephrin complex via both extracellular and intracellular sites. It is likely that the complex consisting of these four molecules plays a critical role in maintaining slit diaphragm.

The expression of Par- 6 and Par- 3 and their interaction with ephrin-B 1 and nephrin were analyzed in a rat nephrotic model induced by the anti-nephrin antibody injection. Proteinuria in this model is caused by disarrangement of the slit diaphragm components. ${ }^{25,37,38}$ In this nephrotic model, the expressions of Par- 6 and ephrin-B 1 decreased quickly just after the disease induction (1 hour). By contrast, no alterations of Par-3 or nephrin were detected in the initiation phase (Figure 4). On day 5 , when abnormal proteinuria was still detected, the expressions of Par- 6 and ephrin-B1 recovered. On the other hand, the expression of Par-3 and nephrin were clearly decreased on day 5. The observations indicated that the change of Par-6 expression accords with that of ephrin-B1, and the change of Par-3 accords with nephrin. The analyses with the nephrotic model also showed that Par-6 is highly associated with ephrin-B1, and Par-3 is highly associated with nephrin. Another important finding, which feels a little strange but is interesting, is that the alteration of Par6-ephrin-B1 advanced the alteration of Par-3-nephrin in the nephrotic model that was induced by the stimulation to nephrin. Dual-labeling analyses in this study showed that the remaining Par- 6 on podocyte at 1 hour of this nephrotic model still had close proximity with ephrin-B1, but it was clearly aside from nephrin and Par-3 (Figure 5). These findings suggest that the down-regulation of Par- 6 and ephrin-B1 and 


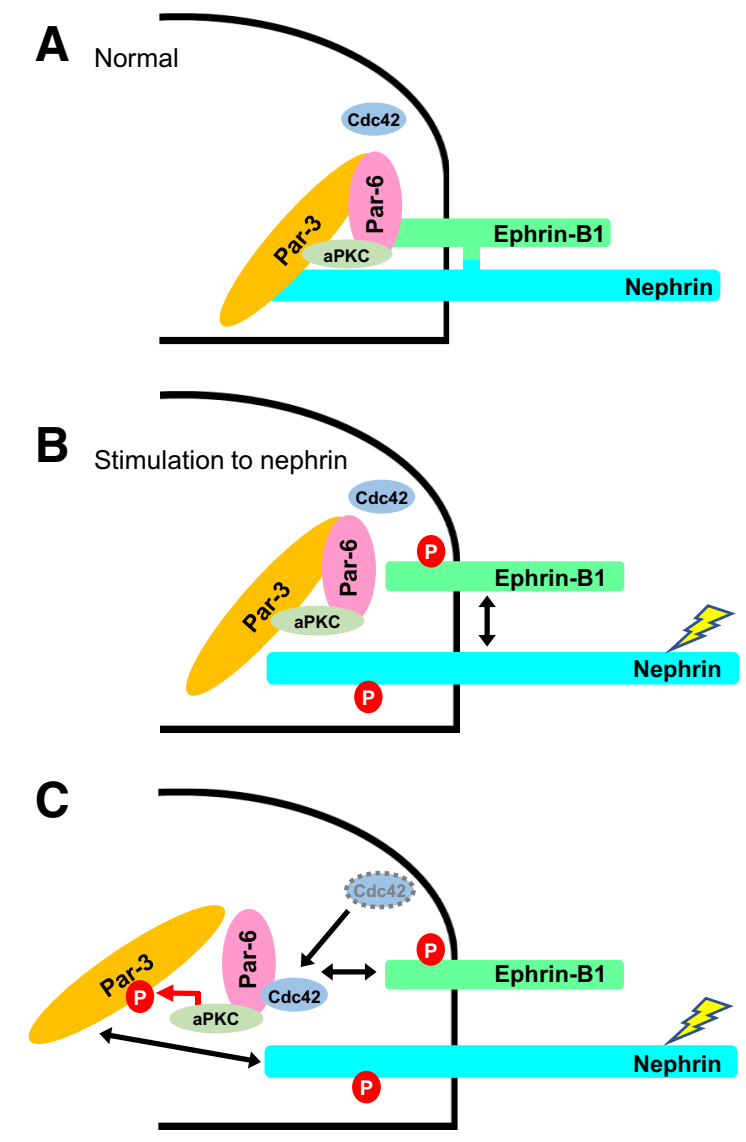

Figure 9 Schematic diagram showing the proposed pathogenic mechanism of disruption of Par-6/ephrin-B1 and Par-3/nephrin complex. A: Par6 interacts with ephrin-B1 but not with nephrin, and Par-3 interacts with nephrin but not with ephrin-B1. Ephrin-B1 interacts with nephrin via their extracellular portions, and Par-6 interacts with Par-3. Atypical protein kinase $\mathrm{C}$ (aPKC) interacts with both Par- 6 and Par-3. The molecular complex plays a critical role in maintaining the slit diaphragm function. B: If the extracellular domain of nephrin is stimulated, ephrin-B1 as well as nephrin is phosphorylated, and the interaction between ephrin-B1 and nephrin was disrupted. C: The phosphorylated nephrin dissociates from Par-3. The phosphorylated ephrin-B1 dissociates from Par-6. Cell division cycle 42 (Cdc42) replaces ephrin-B1 and binds to Par-6. The binding of Cdc42 to Par- 6 increases aPKC activity, the increase of aPKC activity enhances the phosphorylation of Par-3, and the phosphorylated Par-3 departs from Par-6.

the delinking of the Par-6/ephrin-B1 complex from the Par-3/ nephrin complex is an essential initiation event of the slit diaphragm dysfunction.

Nephrin, a key molecule of the slit diaphragm, has a long extracellular domain and serves as a signaling molecule to transmit signals from the slit diaphragm into cytoplasm, although the role of nephrin phosphorylation in pathogenesis of slit diaphragm injury is not well understood yet. ${ }^{41}$ This study shows that not only nephrin but also ephrin-B1 was phosphorylated in the nephrotic model, caused by the injection with the anti-nephrin antibody (Figure 6). The finding showed that ephrin-B1 serves as a signaling molecule to transmit the signals nephrin detected. The interaction of ephrin-B1 with Par-6 was disrupted if ephrin-B1 was phosphorylated. If ephrin-B1 is phosphorylated, the interaction with nephrin is disrupted. ${ }^{13}$ The assay with the HEK cell expression system also showed that the interaction of Par-3 and nephrin was disrupted if nephrin was phosphorylated (Figure 6). These findings suggest that the complex consisting of Par-6, ephrin-B1, Par-3, and nephrin was broken up into separate components in the nephrotic state. The disruption of the complex induced by the nephrinmediated signal is one of the critical mechanisms of the slit diaphragm injury.

In the present study, the developmental expression of Par-6 was also investigated. This is the first report of the developmental study of Par- 6 in podocytes. The expression of Par- 6 was already detected in presumptive podocytes of the early S-shaped body stage, when nephrin did not appear yet and ephrin-B1 staining was faint (Figure 7). At this stage, Par-6 was mainly detected at apical surface area. At the late S-shaped body stage, ephrin-B1 and nephrin staining was detected at the basolateral area, and Par- 6 was broadly detected along almost whole cell surface. Then, Par- 6 became to be restricted to basolateral area of podocytes at the capillary loop stage. Par-3 also already appeared in podocytes of the early S-shaped body stage. Par-3 was mainly expressed at the basolateral area of podocytes at this stage. Major parts of Par3 colocalized with nephrin already at the late S-shaped body stage. The findings on Par-3 observed in this study basically coincide with those of previous reports. ${ }^{21,42}$ These observations implied that Par- 6 appears in early immature podocytes independently of Par-3, ephrin-B1, or nephrin, and that Par-6 moved to basal area to participate in the formation of slit diaphragm at the final stage. It is understood that slit diaphragms appear in podocytes and gradually replace tight junction during the capillary loop stage. ${ }^{43}$ It is plausible that the interaction of Par-6 with ephrin-B1 is a final critical event to complete the formation of slit diaphragm.

To further analyze the significance of the disruption of the complex in podocyte injury, the expression of Par-6 was analyzed in other nephrotic models. The down-regulation of Par-6 at the early phase when proteinuria did not occur yet was observed not only in anti-nephrin antibody-induced nephropathy but also in other nephrotic models, PAN nephropathy, a mimic of MCNS, and ADR nephropathy, a mimic of FSGS (Figure 8). This observation suggests that the decrease of Par-6 expression could be an early sensitive marker to detect podocyte injury. Although the expression of Par-6 already recovered on day 5 when proteinuria was still massive in the anti-nephrin antibody-induced nephropathy, the decrease of Par-6 expression was still evident in proteinuric phase in PAN nephropathy (Figure 8). Morphologic alterations, such as foot process effacement and actin cytoskeletal alteration, were more evident in PAN nephropathy than in the anti-nephrin antibody-induced nephropathy. ${ }^{28,39,40}$ The decrease of Par- 6 expression was prolonged in ADR nephropathy in which persistent proteinuria and severe morphologic alterations were observed. ${ }^{40}$ It is conceivable that the monitoring of Par-6 expression helps us to understand the type and degree of podocyte injury. 
The Par polarity complex of Par-6, Par- 3 , and aPKC plays an essential role for the formation and maintenance of tight junction of epithelial cells. ${ }^{15-17}$ A small GTPase, $\mathrm{Cdc} 42$, is involved in the establishment of polarity in a variety of cell types. ${ }^{16,17,44}$ Active Cdc42 binds to Par-6 and promotes the aPKC activity, and the binding regulates cell polarity in epithelial cells. ${ }^{45-47}$ Ephrin-B1 competes with active $\mathrm{Cdc} 42$ for binding to Par-6, and this competition reduces aPKC activity. ${ }^{14}$ Ephrin-B1 overexpression disrupts tight junctions. The slit diaphragm, a cell-to-cell interaction of podocytes, is a unique cell-cell junction. Although it is still controversial whether the slit diaphragm is a variant of tight junction or of adherence junction, ${ }^{48}$ slit diaphragm is thought to be a variant of tight junction, as it serves both the barrier function and fence functions. ${ }^{43,49-51}$ However, slit diaphragm has some characteristics unlike tight junction (eg, its unique components and a long intercellular distance). Ephrin-B1 is restrictedly expressed at slit diaphragm of podocyte in kidney, and a clear positive staining is not detected at tight junctions of other types of cells in kidney. ${ }^{12}$ Ephrin-B1 is essential for maintaining the molecular integrity and the barrier function of slit diaphragm. ${ }^{12,13}$ This study showed that ephrin-B1 was phosphorylated and down-regulated in the nephrotic models with slit diaphragm dysfunction. Also, the phosphorylated ephrin-B1 did not interact with Par-6. Taken together, we propose the pathogenic mechanism of disruption of Par-6/ephrin-B1 and Par-3/nephrin complex. Schematic diagram of the putative model is shown in Figure 9. In physiologic state, the extracellular components of the slit diaphragm, nephrin and ephrin-B1, are connected to each other via their extracellular domains. The slit diaphragm complex of nephrin and ephrin B1 interacts with the Par complex composed of Par-3, Par6 , and aPKC via their cytoplasmic domain. Nephrin binds to Par-3, and ephrin-B1 binds to Par-6. The link of the slit diaphragm complex with the Par complex is essential for maintaining the barrier function of slit diaphragm. If the extracellular domain of nephrin is stimulated, both nephrin and ephrin-B1 are phosphorylated and the interaction between nephrin and ephrin-B1 is disrupted. Par-3 is dissociated from the phosphorylated nephrin, and Par-6 is dissociated from the phosphorylated ephrin-B1. Cdc42 replaces ephrin-B1 and binds to Par- 6 . The binding of $\mathrm{Cdc} 42$ to Par-6 increases aPKC activity of the Par complex. The increase of aPKC activity induces the phosphorylation of Par-3, and the phosphorylated Par-3 is dissociated from Par-6.

The transition of slit diaphragm to tight junction appears in many nephrotic conditions. ${ }^{52,53}$ It is estimated that the dissociation of Par-6 from ephrin-B1 is an important pathogenic mechanism resulting in the disruption of slit diaphragm and the restoration of tight junction. This study improves our understanding of podocyte biology and the pathogenesis of nephrotic syndrome. Par-6 stability may be therapeutically modulated in treating nephrotic syndromes.

\section{Acknowledgments}

We thank Mutsumi Kayaba and Yukina Kitazawa for excellent technical assistance; Masaaki Nameta for taking electron microscopy images; Dr. Ian Macara for providing pk-myc-Par-6 and pk-myc-Par-3; and Dr. Peter Mundel (Albert Einstein College of Medicine, Bronx, NY) for donating the conditionally immortalized mouse podocyte cell line.

\section{Author Contributions}

S.T. and H.K. designed the experiments; S.T. performed major parts of in vivo experiments and wrote the manuscript; Y.F. performed the studies with conditional knockout mice and in vitro experiments; Y.Z. contributed to the developmental study; I.N. analyzed nephrotic models; H.K. conceived and directed the project and wrote the manuscript.

\section{References}

1. Pavenstadt H, Kriz W, Kretzler M: Cell biology of the glomerular podocyte. Physiol Rev 2003, 83:253-307

2. Holthofer H: Molecular architecture of the glomerular slit diaphragm: lessons learnt for a better understanding of disease pathogenesis. Nephrol Dial Transplant 2007, 22:2124-2128

3. Kawachi H, Shimizu F: Molecular composition and function of the slit diaphragm: nephrin, the molecule responsible for proteinuria. Clin Exp Nephrol 2000, 4:161-172

4. Kestila M, Lenkkeri U, Mannikko M, Lamerdin J, McCready P, Putaala H, Ruotsalainen V, Morita T, Nissinen M, Herva R, Kashtan CE, Peltonen L, Holmberg C, Olsen A, Tryggvason K: Positionally cloned gene for a novel glomerular protein-nephrin-is mutated in congenital nephrotic syndrome. Mol Cell 1998, 1: $575-582$

5. Donoviel DB, Freed DD, Vogel H, Potter DG, Hawkins E, Barrish JP, Mathur BN, Turner CA, Geske R, Montgomery CA, Starbuck M, Brandt M, Gupta A, Ramirez-Solis R, Zambrowicz BP, Powell DR: Proteinuria and perinatal lethality in mice lacking NEPH1, a novel protein with homology to NEPHRIN. Mol Cell Biol 2001, 21:4829-4836

6. Boute N, Gribouval O, Roselli S, Benessy F, Lee H, Fuchshuber A, Dahan K, Gubler MC, Niaudet P, Antignac C: NPHS2, encoding the glomerular protein podocin, is mutated in autosomal recessive steroid-resistant nephrotic syndrome. Nat Genet 2000, 24:349-354

7. Arvanitis D, Davy A: Eph/ephrin signaling: networks. Genes Dev 2008, 22:416-429

8. Davy A, Aubin J, Soriano P: Ephrin-B1 forward and reverse signaling are required during mouse development. Genes Dev 2004, 18: $572-583$

9. Compagni A, Logan M, Klein R, Adams RH: Control of skeletal patterning by ephrinB1-EphB interactions. Dev Cell 2003, 5: $217-230$

10. Tanaka M, Kamata R, Sakai R: Phosphorylation of ephrin-B1 via the interaction with claudin following cell-cell contact formation. EMBO J 2005, 24:3700-3711

11. Ogawa K, Wada H, Okada N, Harada I, Nakajima T, Pasquale EB, Tsuyama S: EphB2 and ephrin-B1 expressed in the adult kidney regulate the cytoarchitecture of medullary tubule cells through Rho family GTPases. J Cell Sci 2006, 119:559-570 
12. Hashimoto T, Karasawa T, Saito A, Miyauchi N, Han GD, Hayasaka K, Shimizu F, Kawachi H: Ephrin-B1 localizes at the slit diaphragm of the glomerular podocyte. Kidney Int 2007, 72:954-964

13. Fukusumi Y, Zhang Y, Yamagishi R, Oda K, Watanabe T, Matsui K, Kawachi H: Nephrin-binding ephrin-B1 at the slit diaphragm controls podocyte function through the JNK pathway. J Am Soc Nephrol 2018, 29:1462-1474

14. Lee HS, Nishanian TG, Mood K, Bong YS, Daar IO: EphrinB1 controls cell-cell junctions through the Par polarity complex. Nat Cell Biol 2008, 10:979-986

15. Joberty G, Petersen C, Gao L, Macara IG: The cell-polarity protein Par6 links Par3 and atypical protein kinase $\mathrm{C}$ to Cdc42. Nat Cell Biol 2000, 2:531-539

16. Lin D, Edwards AS, Fawcett JP, Mbamalu G, Scott JD, Pawson T: A mammalian PAR-3-PAR-6 complex implicated in Cdc42/Rac1 and aPKC signalling and cell polarity. Nat Cell Biol 2000, 2:540-547

17. Noda Y, Takeya R, Ohno S, Naito S, Ito T, Sumimoto H: Human homologues of the Caenorhabditis elegans cell polarity protein PAR6 as an adaptor that links the small GTPases Rac and Cdc42 to atypical protein kinase C. Genes Cells 2001, 6:107-119

18. Hartleben B, Schweizer H, Lubben P, Bartram MP, Moller CC, Herr R, Wei C, Neumann-Haefelin E, Schermer B, Zentgraf H, Kerjaschki D, Reiser J, Walz G, Benzing T, Huber TB: Neph-Nephrin proteins bind the Par3-Par6-atypical protein kinase C (aPKC) complex to regulate podocyte cell polarity. J Biol Chem 2008, 283: 23033-23038

19. Hirose T, Satoh D, Kurihara H, Kusaka C, Hirose H, Akimoto K, Matsusaka T, Ichikawa I, Noda T, Ohno S: An essential role of the universal polarity protein, aPKClambda, on the maintenance of podocyte slit diaphragms. PLoS One 2009, 4:e4194

20. Huber TB, Hartleben B, Winkelmann K, Schneider L, Becker JU, Leitges M, Walz G, Haller H, Schiffer M: Loss of podocyte aPKClambda/iota causes polarity defects and nephrotic syndrome. J Am Soc Nephrol 2009, 20:798-806

21. Hartleben B, Widmeier E, Suhm M, Worthmann K, Schell C, Helmstadter M, Wiech T, Walz G, Leitges M, Schiffer M, Huber TB: aPKClambda/iota and aPKCzeta contribute to podocyte differentiation and glomerular maturation. J Am Soc Nephrol 2013, 24: 253-267

22. Satoh D, Hirose T, Harita Y, Daimon C, Harada T, Kurihara H, Yamashita A, Ohno S: aPKClambda maintains the integrity of the glomerular slit diaphragm through trafficking of nephrin to the cell surface. J Biochem 2014, 156:115-128

23. Committee for the Update of the Guide for the Care and Use of Laboratory AnimalsNational Research Council: Guide for the Care and Use of Laboratory Animals: Eighth Edition. Washington, DC, National Academies Press, 2011

24. Orikasa M, Matsui K, Oite T, Shimizu F: Massive proteinuria induced in rats by a single intravenous injection of a monoclonal antibody. J Immunol 1988, 141:807-814

25. Kawachi H, Koike H, Kurihara H, Yaoita E, Orikasa M, Shia MA, Sakai T, Yamamoto T, Salant DJ, Shimizu F: Cloning of rat nephrin: expression in developing glomeruli and in proteinuric states. Kidney Int 2000, 57:1949-1961

26. Topham PS, Kawachi H, Haydar SA, Chugh S, Addona TA, Charron KB, Holzman LB, Shia M, Shimizu F, Salant DJ: Nephritogenic mAb 5-1-6 is directed at the extracellular domain of rat nephrin. J Clin Invest 1999, 104:1559-1566

27. Miyauchi N, Saito A, Karasawa T, Harita Y, Suzuki K, Koike H, Han GD, Shimizu F, Kawachi H: Synaptic vesicle protein 2B is expressed in podocyte, and its expression is altered in proteinuric glomeruli. J Am Soc Nephrol 2006, 17:2748-2759

28. Otaki Y, Miyauchi N, Higa M, Takada A, Kuroda T, Gejyo F, Shimizu F, Kawachi H: Dissociation of NEPH1 from nephrin is involved in development of a rat model of focal segmental glomerulosclerosis. Am J Physiol Renal Physiol 2008, 295: F1376-F1387
29. Nakatsue T, Koike H, Han GD, Suzuki K, Miyauchi N, Yuan H, Salant DJ, Gejyo F, Shimizu F, Kawachi H: Nephrin and podocin dissociate at the onset of proteinuria in experimental membranous nephropathy. Kidney Int 2005, 67:2239-2253

30. Fukusumi Y, Wakamatsu A, Takashima N, Hasegawa E, Miyauchi N, Tomita M, Kawachi H: SV2B is essential for the integrity of the glomerular filtration barrier. Lab Invest 2015, 95:534-545

31. Macconi D, Bonomelli M, Benigni A, Plati T, Sangalli F, Longaretti L, Conti S, Kawachi H, Hill P, Remuzzi G, Remuzzi A: Pathophysiologic implications of reduced podocyte number in a rat model of progressive glomerular injury. Am J Pathol 2006, 168: $42-54$

32. Suzuki K, Han GD, Miyauchi N, Hashimoto T, Nakatsue T, Fujioka Y, Koike H, Shimizu F, Kawachi H: Angiotensin II type 1 and type 2 receptors play opposite roles in regulating the barrier function of kidney glomerular capillary wall. Am J Pathol 2007, 170: $1841-1853$

33. Takemoto M, Asker N, Gerhardt H, Lundkvist A, Johansson BR, Saito Y, Betsholtz C: A new method for large scale isolation of kidney glomeruli from mice. Am J Pathol 2002, 161:799-805

34. Mundel P, Reiser J, Zuniga Mejia Borja A, Pavenstadt H, Davidson GR, Kriz W, Zeller R: Rearrangements of the cytoskeleton and cell contacts induce process formation during differentiation of conditionally immortalized mouse podocyte cell lines. Exp Cell Res 1997, 236:248-258

35. Verderame M, Alcorta D, Egnor M, Smith K, Pollack R: Cytoskeletal F-actin patterns quantitated with fluorescein isothiocyanate-phalloidin in normal and transformed cells. Proc Natl Acad Sci U S A 1980, 77: $6624-6628$

36. Narisawa M, Kawachi H, Oite T, Shimizu F: Divalency of the monoclonal antibody 5-1-6 is required for induction of proteinuria in rats. Clin Exp Immunol 1993, 92:522-526

37. Kawachi H, Kurihara H, Topham PS, Brown D, Shia MA, Orikasa M, Shimizu F, Salant DJ: Slit diaphragm-reactive nephritogenic MAb 5 1-6 alters expression of ZO-1 in rat podocytes. Am J Physiol 1997, 273:F984-F993

38. Kawachi H, Koike H, Kurihara H, Sakai T, Shimizu F: Cloning of rat homologue of podocin: expression in proteinuric states and in developing glomeruli. J Am Soc Nephrol 2003, 14:46-56

39. Inokuchi S, Shirato I, Kobayashi N, Koide H, Tomino Y, Sakai T: Re-evaluation of foot process effacement in acute puromycin aminonucleoside nephrosis. Kidney Int 1996, 50:1278-1287

40. Suzuki K, Fukusumi Y, Yamazaki M, Kaneko H, Tsuruga K, Tanaka H, Ito E, Matsui K, Kawachi H: Alteration in the podoplaninezrin-cytoskeleton linkage is an important initiation event of the podocyte injury in puromycin aminonucleoside nephropathy, a mimic of minimal change nephrotic syndrome. Cell Tissue Res 2015, 362: 201-213

41. Martin CE, Jones N: Nephrin signaling in the podocyte: an updated view of signal regulation at the slit diaphragm and beyond. Front Endocrinol 2018, 9:302

42. Hartleben B, Widmeier E, Wanner N, Schmidts M, Kim ST, Schneider L, Mayer B, Kerjaschki D, Miner JH, Walz G, Huber TB: Role of the polarity protein Scribble for podocyte differentiation and maintenance. PLoS One 2012, 7:e36705

43. Fukasawa H, Bornheimer S, Kudlicka K, Farquhar MG: Slit diaphragms contain tight junction proteins. J Am Soc Nephrol 2009, 20:1491-1503

44. Nishimura T, Yamaguchi T, Kato K, Yoshizawa M, Nabeshima Y, Ohno S, Hoshino M, Kaibuchi K: PAR-6-PAR-3 mediates Cdc42induced Rac activation through the Rac GEFs STEF/Tiam1. Nat Cell Biol 2005, 7:270-277

45. Yamanaka T, Horikoshi Y, Suzuki A, Sugiyama Y, Kitamura K, Maniwa R, Nagai Y, Yamashita A, Hirose T, Ishikawa H, Ohno S: PAR-6 regulates aPKC activity in a novel way and mediates cell-cell contact-induced formation of the epithelial junctional complex. Genes Cells 2001, 6:721-731 
46. Suzuki A, Yamanaka T, Hirose T, Manabe N, Mizuno K, Shimizu M, Akimoto K, Izumi Y, Ohnishi T, Ohno S: Atypical protein kinase C is involved in the evolutionarily conserved par protein complex and plays a critical role in establishing epithelia-specific junctional structures. J Cell Biol 2001, 152:1183-1196

47. Gao L, Joberty G, Macara IG: Assembly of epithelial tight junctions is negatively regulated by Par6. Curr Biol 2002, 12:221-225

48. Reiser J, Kriz W, Kretzler M, Mundel P: The glomerular slit diaphragm is a modified adherens junction. J Am Soc Nephrol 2000, 11:1-8

49. Schnabel E, Anderson JM, Farquhar MG: The tight junction protein ZO-1 is concentrated along slit diaphragms of the glomerular epithelium. J Cell Biol 1990, 111:1255-1263

50. Kurihara H, Anderson JM, Farquhar MG: Diversity among tight junctions in rat kidney: glomerular slit diaphragms and endothelial junctions express only one isoform of the tight junction protein ZO-1. Proc Natl Acad Sci U S A 1992, 89:7075-7079

51. Kawachi H, Abrahamson DR, St John PL, Goldstein DJ, Shia MA, Matsui K, Shimizu F, Salant DJ: Developmental expression of the nephritogenic antigen of monoclonal antibody 5-1-6. Am J Pathol $1995,147: 823-833$

52. Gong Y, Sunq A, Roth RA, Hou J: Inducible expression of claudin-1 in glomerular podocytes generates aberrant tight junctions and proteinuria through slit diaphragm destabilization. J Am Soc Nephrol 2017, 28:106-117

53. Succar L, Boadle RA, Harris DC, Rangan GK: Formation of tight junctions between neighboring podocytes is an early ultrastructural feature in experimental crescentic glomerulonephritis. Int J Nephrol Renovasc Dis 2016, 9:297-312 